\title{
A Vanishing Theorem for Proper Direct Images
}

\author{
By \\ Takeo OHSAWA*
}

\section{Indrodustion}

Let $X$ be a complex analytic space and $\mathscr{F}$ a coherent analytic sheaf over $X$. Jf $X$ is $q$-complete, then $H^{r}(X, \mathscr{F})$, the $r$-th cohomology of $X$ with coefficients in $\mathscr{F}$, vanishes for $r \geq q$ (cf. Andreotti-Grauert [1]). If moreover $X$ is a $q$-complete manifold of dimension $n$ and $\mathcal{F}$ is locally free, then one has $H_{0}^{n-r}\left(X, \omega_{X} \otimes \mathscr{F}\right)=0$ by Serre's duality, where $\omega_{X}$ denotes the canonical sheaf of $X$ and $H_{0}^{n-r}$ denotes the cohomology with compact supports.

In [2], A. Andreotti and E. Vesentini established an $L^{2}$-theory on noncompact complex manifolds and showed that the vanishing of $H_{0}^{n-r}\left(X, \omega_{X} \otimes \mathscr{F}\right)$ is a direct consequence of certain a priori estimate as well as the vanishing of $H^{r}(X, \mathscr{F})$. Their approach is of interest since the cohomology vanishing is reduced to the solvability of a $\bar{\partial}$-equation with uniform estimates related to weighted $L^{2}$-norms of Carleman type.

The purpose of the present article is to give a relative version of their theory in the following situation.

Let $f: X \rightarrow S$ be a morphism between complex analytic spaces and $\mathscr{F}$ a coherent analytic sheaf over $X$. The $q$-th direct image sheaf $R^{q} f_{*} \mathscr{F}$ is a sheaf over $S$ defined by $\left(R^{q} f_{*} \mathscr{F}\right)_{x}:=\lim _{\sigma} H^{q}\left(f^{-1}(U), \mathscr{F}\right)$, where $U$ runs through the neighbourhoods of $x$. The $q$-th proper direct image sheaf $R^{q} f_{1} \mathscr{F}$ is defined by $\left(R^{q} f_{!} \mathscr{F}^{\prime}\right)_{x}:=\lim _{\sigma} H_{\mathscr{Q}}^{q}\left(f^{-1}(U), \mathscr{F}\right)$, where $\mathscr{L}$ denotes the family of supports consisting of the subsets of $X$ on which $f$ is proper.

A recent result of $\mathbb{K}$. Takegoshi shows that $R^{q} f_{*} \omega_{X}=0$ for $q>0$ in the above situation, if $X$ is a complex manifold which is bimeromorphically equivalent to a Stein space.

Received April 28, 1986.

* Research Institute for Mathematical Sciences, Kyoto University, Kyoto 606, Japan. 
Similarly as above, Takegoshi's vanishing theorem implies that $\mathbb{R}^{q} f_{!} \Theta_{X}$ $=0$ for $q<\operatorname{dim} X-\operatorname{dim} S$, by the duality theorem of Ramis and Ruget (cf. [9]).

Thus our task here shall be to give a direct proof to $R^{q} f_{!} \mathcal{O}_{X}=0$ in the spirit of Andreotti-Vesentini. It will be reduced to finding two convex increasing functions on $\mathbb{R}$ which compose the weight functions controling the supports of the solutions of the $\bar{\partial}$-equation.

The author expresses his sincere thanks to Professor T. Oda for the stimulating discussion, and to Professor J. Kollar for drawing the author's attention to Ramis-Ruget's duality. Last but not least he thanks to the referee for pityless criticisms.

\section{§1. $\mathbb{L}^{2} \cdot$ Colnømology Vanishing}

Let $\left(X, d s^{2}\right)$ be a Hermitian manifold of dimension $n$ and $(E, h)$ a holomorphic Hermitian vector bundle over $X$. We denote by $L_{\mathrm{loc}}^{*}(E)$ the set of locally square integrable differential forms on $X$ with values in $E$, and by $L_{\mathrm{loc}}^{p, q}(E)$, or by $L_{\mathrm{loc}}^{p, q}(X, E)$, the set of $(p, q)$-forms in $L_{\mathrm{loc}}^{*}(E)$. For any $C^{\infty}$ function $\psi: X \rightarrow \mathbb{R}$ we denote by $L^{*}(E)$ the set of $\mathcal{U} \in L_{\mathrm{loc}}^{*}(E)$ with

$$
\int_{X} e^{-\psi}|u|^{2} d V<\infty
$$

Here $|u|$ denotes the length of $u$ and $d V$ the volume form. We put

$$
\|u\|_{\psi:}:=\left\{\int_{X} e^{-\psi}|u|^{2} d V\right\}^{1 / 2}
$$

Then $L^{*}(E)$ is a Hilbert space with norm \|\|$_{\psi}$. We put $L_{\psi}^{p, q}(E)$ $\left(=L_{\psi}^{p, q}(X, E)\right):=L_{10 c}^{p, q}(E) \cap L_{\psi}^{*}(E)$. Let $\langle u, v\rangle$ be the pointwise inner product of $u$ and $v$ in $L_{\mathrm{loc}}^{*}(E)$ with respect to the metrics $d s^{2}$ and $h$. Then the inner product of $u$ and $v$ is expressed as

$$
(u, v)_{\psi}:=\int_{X} e^{-\psi}\langle u, v\rangle d V
$$

The complex differentiation $\bar{\partial}: L_{1 \mathrm{oc}}^{*}(E) \rightarrow L_{\mathrm{loc}}^{*}(E)$ is defined in the sense of distribution. The domain of the restriction of $\bar{\partial}$ to $I^{*}(E)$ will be denoted by $D_{\bar{\partial}}$, shortly. Clearly $\bar{\partial}$ is a densely defined closed linear operator for any $\psi$. We denote by $\bar{\partial}_{\psi}^{*}$ the adjoint of $\vec{\partial}$ on $L_{\psi}^{*}(E)$. The domain of $\bar{\partial} *$ is denoted by $D_{\bar{\partial}}^{*}$. The following is a direct consequence of Hahn-Banach's theorem. For the proof, the reader is referred to Hormander's book [6] (cf. 
Chap. IV).

$\mathbb{P r}_{0} 0$ position $\mathbb{H}_{0} \mathbb{1}$ For any $v \in L_{\psi}^{*}(E)$ the following conditions (i) and (ii) are equivalent.

(i) $\left|(u, v)_{\psi}\right| \leq C\left\|\bar{\partial}_{\psi}^{*} u\right\|_{\psi}$, for any $u \in D_{\bar{\partial}}^{*}$ with $\bar{\partial} u=0$.

(ii) There exists $a w \in L_{\psi}^{*}(E)$ satisfying $\bar{\partial} w=v$ and $\|w\|_{\psi} \leq \mathbb{C}$.

Here $\mathbb{C}$ is a positive number which depends on $v$.

To study geometric conditions which imply the condition (i) above, the following lemma is helpful.

Lemma 1.2 (cf. [2]) Let $\left(X, d s^{2}\right)$ be a complete Hermitian manifold, $(E, h)$ a Hermitian vector bundle over $X, A: L_{\psi}^{*}(E) \rightarrow L_{\psi}^{*}(E)$ a bounded linear operator, and $(p, q)$ a pair of nonnegative integers. If the inequality $\|A u\|_{\psi}^{2} \leq\|\bar{\partial} u\|_{\psi}^{2}+$ $\|\bar{\partial} * u\|_{\psi}^{2}$ holds for any compactly supported $E$-valued $C^{\infty}(p, q)$-form $u$ on $X$, then the same inequality is valid for all $u \in D \bar{\partial} \cap D_{\bar{\partial}}^{*} \mathcal{L} \cap_{\psi}^{p, q}(E)$. Moreover, for any $v \in \operatorname{Im} A$, (i) holds with $C=\left\|A^{-1} v\right\|_{\psi}$.

Proof. For the first part, see [2]. The second assertion follows from Cauchy-Schwartz inequality.

Before proceeding further, we explain the notion of Nakano seminegativity of the bundle $(E, h)$.

By identifying the metric $h$ with a $C^{\infty}$ section of $\operatorname{Hom}\left(E, \bar{E}^{*}\right)$, we define the curvature of $(E, h)$ by $\bar{\partial} \circ h^{-1} \circ \partial \circ h$, which is naturally identified with a $\mathbb{C}^{\infty}$ section, say $\Theta_{h}$, of the bundle $T_{X}^{*} \otimes \bar{T}_{X}^{*} \otimes \operatorname{Hom}(E, E)$ or equivalently, of the bundle $\operatorname{Hom}\left(T_{X}, \bar{T}_{X}^{*}\right) \otimes \operatorname{Hom}(E, E)$, where $T_{X}$ denotes the holomorphic tangent bundle of $X$. Thus $h \circ \bar{\partial} \circ h^{-1} \circ \partial \circ h$ is naturally identified with a section of $\operatorname{Hom}\left(E \otimes T_{X}, \bar{E}^{*} \otimes \bar{T}_{X}^{*}\right)$, which defines a quadratic form along the fibers of $E \otimes T_{X}$.

Definnition. A Hermitian vector bundle $(E, h)$ is said to be Nakano seminegative if $h \circ \bar{\partial} \circ h^{-1} \circ \partial \circ h$ is a negative semidefinite quadratic form on $F \otimes T_{X}$.

In what follows we shall regard $\Theta_{h}$ as a $(1,1)$-form with values in $\operatorname{Hom}(E, E)$.

Lemama $\mathbb{1 . 3}$ Let $\left(X, d s^{2}\right)$ be a Kahler manifold of aimension $n,(E, h)$ a Nakano seminegative vector bundle over $X$, and $\psi$ a $\mathbb{C}^{\infty}$ real valued function on $X$. Let $r_{1} \geq \cdots \geq r_{n}$ be the eigenvalues of $i \partial \bar{\partial} \psi$ on $X$. Then, $\|\bar{\partial} u\|_{\psi}^{2}+\left\|\bar{\partial} \psi_{\psi}^{*} u\right\|_{\psi}^{2}$ $\geq\left(-\left(r_{1}+\cdots+r_{n-q}\right) u, u\right)$, for any compactly supported E-valued $C^{\infty}(0, q)$-form $u$ on $X$. 
Proof. By Bochner-Nakano's inequality,

$$
\|\bar{\partial} u\|_{\psi}^{2}+\left\|\bar{\partial}_{\psi}^{*} u\right\|_{\psi}^{2} \geq\left(-i \Lambda\left(\left(\Theta_{h}+\partial \bar{\partial} \psi\right) \wedge u\right)_{,} u\right)_{\psi},
$$

for any compactly supported $C^{\infty}(0, q)$-form $u$. Here $\mathbb{1}$ denotes the adjoint of the wedge multiplication by the fundamental form of $d s^{2}$. Since $(E, h)$ is Nakano seminegative, $\left(-i \Lambda\left(\Theta_{h} \wedge u\right), u\right) \geq 0$ (cf. [7] p. 197 (14)). The rest is a straightforward computation.

Theorem $\mathbb{1}_{0} 4$ Let $(E, h)$ be a Nakano seminegative Hermitian vector bundle over a complex manifold $X$ of dimension $n$, let $\Phi_{1}, \Phi_{2}$ be two $C^{\infty}$ plurisubharmonic functions on $X$ and let $r$ be an integer such that rank $\partial \bar{\partial} \Phi_{2} \leq r$ everywhere. Suppose that $i \partial \bar{\partial}\left(\Phi_{1}+\Phi_{2}\right)$ is a positive $(1,1)$-form associated to a complete Kahler metric on $X$, say $d s^{2}$. Then, for any $q<n-r$ and $v \in L_{-(r+1) \Phi_{1}+\Phi_{2}}^{0, q}(E)$ with $\vec{\partial} v=0$, where the metric on $X$ is $d s^{2}$, there exists $a w \in L_{-(r+1) \Phi_{1}+\Phi_{2}}^{0, q-1}(E)$ such that $\bar{\partial} w=v$ and $\|w\|_{-(r+1) \Phi_{1}+\Phi_{2}} \leq\|v\|_{-(r+1) \Phi_{1}+\Phi_{2}}$. Here we use a convention that $L_{\psi}^{0, q}(E)=0$ for $q<0$.

Proof. Let $\Gamma_{1} \geq \cdots \geq \Gamma_{n}$ be the eigenvalues of $i \partial \bar{\partial}\left(-(r+1) \Phi_{1}+\Phi_{2}\right)$ with respect to the metric $d s^{2}\left(=2 \partial \bar{\partial}\left(\Phi_{1}+\Phi_{2}\right)\right)$. By assumption, $-\left(\Gamma_{1}+\cdots+\Gamma_{n-q}\right)$ $\geq 1$ everywhere on $X$. Hence the assertion follows from Proposition 1.1, Lemma 1.2 and Lemma 1.3.

In the following paragraph we shall prove the following.

Theorem Let $X$ be a complex manifold of pure dimension $n$ which is bimeromorphically equivalent to a Stein space, $(E, h)$ a Nakono seminegative bundle over $X, S$ a complex space of dimension $s$, and $f$ a holomorphic map from $X$ to $S$. Then $R^{q} f_{1} \Theta_{X}(E)=0$ for $q<n-s$.

Since every holomorphic vector bundle admits a Nakano seminegative Hermitian structure on Stein manifolds, we have the following corollary.

Corollary Let $X$ be a Stein manifold of pure dimension $n, S$ a complex analytic space of dimension $s$, and $f: X \rightarrow S$ a holomorplic map. Then, for any holomorphic vector bundle E. over $X, R^{q} f_{1} \Theta_{X}(E)=0$ for $q<n-s$.

\section{Proof of Theorem}

1. The Stein case: First we shall give the proof when $X$ is a Stein manifold because it is completely independent of the other results which we need in the proof of the general case. Assume that we are given a Stein manifold 
$X$ of pure dimension $n$, a Nakano seminegative vector bundle $(E, h)$ over $X$, a complex space $S$ of dimension $s$ and a holomorphic map $f: X \rightarrow \mathbb{S}$. Let $q$ be an integex with $q<n-s$ and $v \in L_{10 c}^{0, p}(E)$ a $\bar{\partial}$-closed form such that $f \mid \operatorname{supp} v$ is proper. Since the problem is local on $S$, we may assume that $S$ is a Stein space and that there exists a $\mathbb{C}^{\infty}$ strictly plurisubharmenic function $\Phi_{1}$ on $X$ such that supp $v \subset X_{c}:=\left\{x \in X ; \Phi_{1}(x) \leq c_{0}\right\}$ for some $c_{0} \in \mathbb{R}$ and the restriction of $f$ to $X_{c}$ is proper for all $c \in \mathbb{R}$. Let $\varphi_{\circ}$ be a $\mathbb{C}^{\infty}$ plurisubharmonic exhaustion function on $S$ and set $\varphi:=\varphi_{\circ} \circ f$. Evidently, there exists a $\mathbb{C}^{\infty}$ convex increasing furction $\lambda: \mathbb{R} \rightarrow \mathbb{R}$ such that the metric $d s^{2}:=2 \partial \bar{\partial}\left(\Phi_{1}+\lambda(\varphi)\right)$ is complete and that $v \in \mathbb{L}_{-(s+1) \Phi_{1}+\lambda(\varphi)}^{0, q}(E)$ (with respect to $d s^{2}$ ). Eet $\chi$ be a $C^{\infty}$ convex increasing function on $\mathbb{R}$ such that $\chi(t)=0$ for $t \leq c_{0}$ and $\chi^{\prime}(\hat{t})=1$ for $t \geq c_{0}+1$. Clearly $\|v\|_{-(s+1) \Phi_{1}+\lambda(\varphi)-\mu_{\chi}\left(\Phi_{1}\right)}=\|v\|_{-(s+1) \Phi_{1}+\lambda(\varphi)}$ for any $\mu>0$. Since $\partial \bar{\partial} \chi\left(\Phi_{1}\right) \geq 0$ by Theorem 1.4 one can find a $w_{\mu} \in \mathbb{L}_{-(s+1) \Phi_{1}+\lambda(\varphi)-\mu_{\chi}\left(\Phi_{1}\right)}^{0, q-1}(E)$ satisfying $\bar{\partial} w_{\mu}=v$ and $\left\|w_{\mu}\right\|_{-(s+1) \Phi_{1}+\lambda(\varphi)-\mu_{x}\left(\Phi_{1}\right)} \leq\|v\|_{-(s+1) \Phi_{1}+\lambda(\varphi)}$, for any $\mu>0$.

If $\mu \geqslant \nu$ one has

$$
\left\|w_{u}\right\|_{-(s+1) \Phi_{1}+\lambda(\varphi)-\mu_{x}\left(\Phi_{1}\right)} \geq\left\|w_{\mu}\right\|_{-(s+1) \Phi_{1}+\lambda(\varphi)-v x\left(\Phi_{1}\right)} .
$$

Therefore one can choose a subsequence of $\left\{w_{\mu}\right\}_{\mu=1}^{\infty}$ which converges weakly to some $w \in L_{10 c}^{0, q-1}(E)$. Clearly $\bar{\partial}^{\prime}=v$ and supp $w \subset X_{c_{0}}$, which implies that $v$ represents the 0 -class in $\mathbb{H}_{\mathscr{Q}}^{q}\left(X, \mathcal{O}_{X}(E)\right)$ with $\mathscr{L}:=\{A \subset X ; f \mid A$ is proper .

2. General case. To prove our theorem in full generality we rely upon the desingularization theorem of Hironaka in the following form (cf [5]).

Theorem 2.11 Let $Y$ be a Stein space of dimension $n$ and let $X$ be o complex space with a proper holomorphic map $\pi: X \rightarrow Y$ such that $\pi \mid X \backslash \pi^{-1}$ (Sing $Y$ ) is biholomorphic. Here Sing $Y$ denotes the singular locus of $Y$. Then, for any point $y \in Y$, there exist a neighbourhood $U \ni y$ and a complex manifold

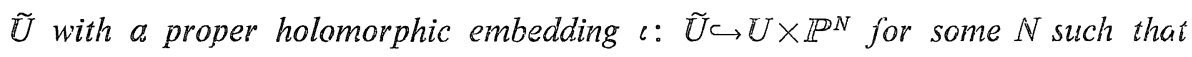
the composite of $\&$ with the projection to the first component factors through $\pi$. i.e., there exists a holomorphic map $p: \widetilde{U} \rightarrow \pi^{-1}(U)$ such that the diagram

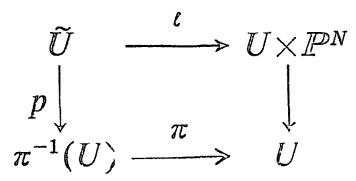

comnites.

Similarly as in Proposition 4.1 in [8], we may apply Hironaka's theorem to obtain the following: 
Proposition 2.2 Let $X$ be a complex manifold of pure dimension $n$ bimeromorphic to a Stein space and let $\pi: X \rightarrow \hat{X}$ be its Remmert reduction. Then, for any point $x \in \hat{X}$, there exist a neighbourhood $U$, an ancilytic subset $A \subset \pi^{-1}(U)$ and a plurisubharmonic function $\psi$ on $\pi^{-1}(U$; with $A=\{x ; \psi(x)=-\infty\}$ which satisfies the following properties:

(i) $\psi$ is $C^{\infty}$ outside $A$ and $2 \partial \bar{\partial} \psi$ gives a complete Kahler metric on $\pi^{-1}(U) \backslash A$.

(ii) For any open set $V \Subset \pi^{-1}(U)$ a holomorphic function $h$ (resp. a holomorphic n-form $\omega$ ) on $V \backslash A$ is holomorphically extendable to $V$ if and only if $h \in L_{-\psi}^{0,0}(V \backslash A)$ (resp. $\omega \in L_{\psi}^{n, 0}(V \backslash A)$ ) with respect to the metric $2 \partial \bar{\partial} \psi$.

(iii) For anv $V \Subset \pi^{-1}(U)$ and any holomorphic $n$-form $\omega$ on $V$, the multiplication

$$
\omega \wedge: L_{-\psi}^{0, q}(V \backslash A) \rightarrow L_{\psi}^{n, q}(V \backslash A)
$$

is continuous for all $q \geq 0$.

We shall omit the proof, because the construction of $\psi$ is quite similar to that in [8] (cf. the function $K \varphi^{2}-\log (1+\rho \log \psi$ ) in Proposition 4.1) and the properties above are immediate from the definition of $\psi$.

Our theorem is now contained in the following

Theorem 2.3 Let $X$ be a complex manifold of pure dimension $n,(E, h)$ a Nakano seminegative vector bundle over $X, A \subset X$ an analytic subset, and $\psi$ a plurisubharmonic function satisfying (i), (ii) and (iii) in Proposition 2.2 for $X=\pi^{-1}(U)$. Let $\Psi$ and $\varphi$ be $C^{\infty}$ plurisubharmonic functions on $X$ such that $\Psi+\varphi$ is exhausting. Assume that rank $\partial \bar{\partial} e^{\varphi} \leq s$ everywhere. Then, with respect to the family of supports $\mathscr{Q}:=\left\{U: \sup _{U} \Psi<\infty\right\}$,

$$
H_{\mathscr{P}}^{q}\left(X, \mathcal{O}_{X}(E)\right)=0, \quad \text { for } q<n-s .
$$

Proof. First we assert that it suffices to show that for any $q<n-s$ and a $C^{\infty}(0, q)$-form $v$ on $X$ with $\bar{\partial} v=0$ and supp $\imath \subset\{x ; \Psi(x) \leq c\}$, there exists a $w \in L_{1 \mathrm{oc}}^{0, q-1}(X \backslash A, E)$ with the following properties:

(3) For any point $x \in X$ there exists a neighbourhood $U$ such that $w \in L_{-\psi}^{0, q-1}(U \backslash A, E)$ with respect to the metric $2 \partial \bar{\partial} \psi$.

To see the validity of this assertion, let $\mathcal{U}$ be a locally finite covering of $X$ ky relatively compact polydiscs such that $\sup \left\{\left|\Phi_{1}(x)-\Phi_{1}(y)\right| ; x, y \in U\right\}<1$ 
for any $U \in \mathcal{U}$. Let $\sigma$ be a Čech $q$-cocycle associated to $\mathcal{U}$ such that supp $\sigma \subset\{x ; \Psi(x) \leq c\}$ for some $c$. Then, in a canonical way one can associate to $\sigma$ a $\bar{\partial}$-closed $C^{\infty}(\mathbb{C}, q)$-form $v$ on $X$ such that $\bar{\partial} v=0$ and supp $v \subset\{x ; \Psi(x) \leq c\}$. Suppose that there exists a i' satisfying (1): (2), and (3). Then, from (iii) we can regard $w$ locally as an element of $L_{\psi}^{n, q-1}(U \backslash A, E)$ for each $U \in \mathcal{U}$, by multiplying a holomorphic $n$-form $\omega_{u}$ with nc zero on $\bar{U}$. Hence, applying repeatedly the $L^{2}$-vanishing theorem on $U \backslash A$ (cf. Theorem 2.8 in [3]) we arrive at a Čech $(q-1)$-cochain $\tau$ associated to $\mathcal{U}^{\prime}:=\{U \backslash A\} U \in \mathcal{U}$ such that $\delta \tau=\sigma \mid X \backslash A, \tau_{1 \cdots q} \omega_{U_{1}} \in \mathbb{L}_{\psi}^{n, 0}\left(U_{1} \cap \cdots \cap U_{q} \backslash A, E\right)$ for any $U_{1}, \cdots, U_{q} \in \mathcal{U}$ and that supp $\tau \subset\{x \in X \backslash A ; \Psi(x)<c+1\}$. Hence, by (ii) all $\tau_{1 \cdots q}$ are holomorphically extendable on $U_{1} \cap \cdots \cap U_{q}$. Thus $\sigma$ represents the 0 -class in $\mathbb{H}_{\mathcal{Q}}^{q}\left(X, \mathcal{O}_{X}(E)\right)$.

Now let $v$ be as above and apply Theorem 1.4 as before by letting $\Phi_{1}=\psi+\mu \chi(\Psi), \Phi_{2}=\lambda(\varphi)$ and $d s^{2}=2 \partial \bar{\partial}(\psi+\lambda(\varphi))$, where $\lambda$ is chosen as before Letting $\mu \rightarrow \infty$ we obtain a $w \in L_{10 \mathrm{co}}^{0, q-1}(X \backslash A, E)$ satisfying (1), (2) and (3) abcve.

q.e.d.

Remark Our proof of the cohomology vanishing is direct in the sense it is independent of the coherency theorem for the direct image sheaves (cf. Grauert [4] Siu [10] and Ermine [3]). Moreover it gives a slightly more general result. In fact, in Theorem 2.3, A need not be holomorphically convex. For instance one may take as $A$ a pseudoconvex neighbourhood of the zero section of any topologically trivial line bundle over a compact Kahler manifold.

\section{TReferences}

[1] Andreotti, A. and Grauert, H., Théorème de finitude pour la cohomologie des espace complexes, Bull. Soc. Math. France, 90 (1962) 193-259.

[2] Andreotti, A. and Vesentini, E., Carleman estimates for the Laplace-Belirami equation on complex manifolds, Pvbl. Math. I.H.E.S. 25 (196j) 81-130.

[3] Ermine, J.L., Cohérence de certaines images directes à supports propres dans le cas d'un morphisme fortement p-convexe, Ann. Sc. Norm. Sup. Pisa 6 (1979) 1-18.

[4] Grauert, H., Ein Theorem der analytischen Garbentheorie und die Modulraume komplexer Strukturen, Publ. Math. I.H.E.S. 5 (1960) 233-292.

[5] Hironaka, H., Flattering theorems in complex-analytic geometry, Amer. J. Math. 97 (1975) 503-547.

[6] Hörmander, L., An Introduction to Connjlex Analysis in Several Complex Variables, North-Holland, Amsterdam, 1973.

[ 7 ] Ohsawa. T., Isomorphism theorems for cohomology groups on weakly 1-complete manifolds, Putl. RIMS, Kyoto Univ. $\mathbb{1 8}$ (1982) 191-232.

181 - Vanishing theorems on complcte Kahler manifolds, Publ. RIMS, Kyoto Univ., 20 (1984) 21-38.

[9] Ramis, J.P. and Ruget, G., Residus et dualité, invent. Math. 26 (1974) 82-131. 
[10] Siu, Y.T., The 1-convex generalization of Grauert's direct image theorem, Math. Ann. 190 (1971) 203-214.

[11] Takegoshi, K., Relative vanishing theorems in analytic spaces, Duke Math. J. 52 (1985) 273- 279. 